\title{
Sampling Part Sizes of Random Integer Partitions
}

\author{
Ljuben Mutafchiev \\ American University in Bulgaria, 2700 Blagoevgrad, Bulgaria \\ and Institute of Mathematics and Informatics of the \\ Bulgarian Academy of Sciences \\ e-mail: ljuben@aubg.bg; tel.: +35973 888498
}

\begin{abstract}
Let $\lambda$ be a partition of the positive integer $n$, selected uniformly at random among all such partitions. Corteel et al. (1999) proposed three different procedures of sampling parts of $\lambda$ at random. They obtained limiting distributions of the multiplicity of the randomly-chosen part as $n \rightarrow \infty$. This motivated us to study the asymptotic behavior of the part size under the same sampling conditions. A limit theorem whenever the part is selected uniformly at random among all parts of $\lambda$ (i.e., without any size bias) was proved earlier by Fristedt (1993). We consider the remaining two (biased) procedures and show that in each of them the randomly-chosen part size, appropriately normalized, converges in distribution to a continuous random variable. It turns out that different sampling procedures lead to different limiting distributions.
\end{abstract}

Key words: integer partitions, part sizes, sampling, limiting distributions

Mathematics Subject classifications: 05A17, 60C05, 60F05

\section{Introduction}

Partitioning integers into summands (parts) is a subject of intensive research in combinatorics, number theory and statistical physics. If $n$ is a positive integer, then by a partition, $\lambda$, of $n$, we mean a representation

$$
\lambda: \quad n=\sum_{j=1}^{n} j m_{j},
$$

in which $m_{j}$, called multiplicities of parts $j, j=1,2, \ldots, n$, are non-negative integers. We use $\Lambda(n)$ to denote the set of all partitions of $n$ and let $p(n)=\mid$ $\Lambda(n) \mid$. The number $p(n)$ is determined asymptotically by the famous partition 
formula of Hardy and Ramanujan [8]:

$$
p(n) \sim \frac{1}{4 n \sqrt{3}} \exp \left(\pi \sqrt{\frac{2 n}{3}}\right), \quad n \rightarrow \infty .
$$

A precise asymptotic expansion for $p(n)$ was found later by Rademacher [13] (more details may be also found in [2; Chapter 5]). Further on, we assume that, for fixed integer $n \geq 1$, a partition $\lambda \in \Lambda(n)$ is selected uniformly at random (uar). In other words, we assign the probability $1 / p(n)$ to each $\lambda \in \Lambda(n)$. In this way, each numerical characteristic of $\lambda$ can be regarded as a random variable defined on $\Lambda(n)$.

Corteel et al. [3] proposed and studied three procedures of sampling parts of a random partition $\lambda \in \Lambda(n)$. They focused on the multiplicities $\mu_{n, j}=$ $\mu_{n, j}(\lambda), j=1,2,3$, of the randomly-selected parts and found their limiting distributions, as $n \rightarrow \infty$, in these three cases of sampling.

Our aim in this paper is to determine asymptotically, as $n \rightarrow \infty$, the distributions of the sizes $\sigma_{n, j}=\sigma_{n, j}(\lambda)$ of the randomly-chosen parts for the same sampling procedures. One of these three limiting distributions is obtained earlier by Fristedt [5]. We present the proofs of the other two limit theorems in Sections 4 and 6 . They combine probabilistic and analytical techniques, which are briefly described in Section 3. The main results are also stated there. Sections 2 and 4 contain some definitions, notations and auxiliary facts.

\section{Preliminaries}

We start with the notation $g(x)$ for the generating function of the sequence $\{p(n)\}_{n \geq 1}$. For $|x|<1, g(x)$ admits the well known representation

$$
g(x)=1+\sum_{n=1}^{\infty} p(n) x^{n}=\prod_{k=1}^{\infty}\left(1-x^{k}\right)^{-1}
$$

(see e.g. [2; Theorem 1.1]).

For any $\lambda \in \Lambda(n)$ selected uar, we define the random variables

$$
\alpha_{j}^{(n)}=\alpha_{j}^{(n)}(\lambda)=\text { the number of parts of size } j \text { in } \lambda
$$

and

$$
\beta_{j}^{(n)}=\beta_{j}^{(n)}(\lambda)=\left\{\begin{array}{lll}
1 & \text { if } & \alpha_{j}^{(n)}>0 \\
0 & \text { if } & \alpha_{j}^{(n)}=0 .
\end{array}\right.
$$

Then, obviously, $Z_{n}=\sum_{j=1}^{n} \alpha_{j}^{(n)}$ equals the total number of parts and $Y_{n}=$ $\sum_{j=1}^{n} \beta_{j}^{(n)}$ - the number of distinct parts in $\lambda \in \Lambda(n)$. Furthermore, for $s$ real and $\geq 1$, we also let

$$
X_{s, n}=\left\{\begin{array}{lll}
\sum_{1 \leq j \leq s} j \alpha_{j}^{(n)} & \text { if } & 1 \leq s \leq n, \\
n & \text { if } & s>n ;
\end{array}\right.
$$




$$
\begin{aligned}
& Z_{s, n}=\left\{\begin{array}{lll}
\sum_{1 \leq j \leq s} \alpha_{j}^{(n)} & \text { if } & 1 \leq s \leq n, \\
Z_{n} & \text { if } & s>n ;
\end{array}\right. \\
& Y_{s, n}=\left\{\begin{array}{lll}
\sum_{1 \leq j \leq s} \beta_{j}^{(n)} & \text { if } & 1 \leq s \leq n, \\
Y_{n} & \text { if } & s>n .
\end{array}\right.
\end{aligned}
$$

Next, by $\mathbb{E}(C)$ we denote the expected value of a random variable $C$ with respect to the uniform probability measure $\operatorname{Pr}($.$) defined on the integer partition$ space $\Lambda(n)$. The following two asymptotic equivalences are well known:

$$
\begin{gathered}
\mathbb{E}\left(Y_{n}\right) \sim \frac{\sqrt{6 n}}{\pi}, \quad n \rightarrow \infty, \\
\mathbb{E}\left(Z_{n}\right) \sim \frac{\sqrt{6 n}}{2 \pi} \log n, \quad n \rightarrow \infty
\end{gathered}
$$

(2.7) was proved by Wilf in [15]; a proof of (2.8) can be found in [3]).

We introduce now the joint probability generating functions

$$
\begin{aligned}
& \mathbb{E}\left(y_{1}^{\alpha_{1}^{(n)}} y_{2}^{\alpha_{2}^{(n)}} \ldots y_{n}^{\alpha_{n}^{(n)}}\right)= \\
& \sum_{\sum j m_{j}=n, m_{j} \in \mathbb{N}_{0}} \operatorname{Pr}\left(\alpha_{1}^{(n)}=m_{1}, \alpha_{2}^{(n)}=m_{2}, \ldots, \alpha_{n}^{(n)}=m_{n}\right) \\
& \times y_{1}^{m_{1}} y_{2}^{m_{2}} \ldots y_{n}^{m_{n}},
\end{aligned}
$$

where $\mathbb{N}_{0}=\{0,1, \ldots\}$. General enumeration methods imply that

$$
1+\sum_{n=1}^{\infty} x^{n} p(n) \mathbb{E}\left(y_{1}^{\alpha_{1}^{(n)}} y_{2}^{\alpha_{2}^{(n)}} \ldots y_{n}^{\alpha_{n}^{(n)}}\right)=\prod_{k=1}^{\infty} \sum_{m_{k} \in \mathbb{N}_{0}}\left(y_{k} x^{k}\right)^{m_{k}}
$$

(for more details and proof of (2.10), see [14; Chapter V.5]). Here $x$ and $y_{k}$ are formal variables. Setting in (2.9) and (2.10) $y_{k}=y^{k}$ for $1 \leq k \leq[s]$ and $y_{k}=1$ for $k>[s]([s]$ denotes the integer part of $s)$, from (2.1) and (2.4) we obtain

$$
1+\sum_{n=1}^{\infty} p(n) x^{n} \mathbb{E}\left(y^{X_{s, n}}\right)=g(x) \prod_{1 \leq j \leq s} \frac{1-x^{j}}{1-(x y)^{j}} .
$$

A differentiation with respect to $y$ in this identity leads to the formula for the expectations we want:

$$
\sum_{n=1}^{\infty} p(n) x^{n} \mathbb{E}\left(X_{s, n}\right)=g(x) h_{s}(x),
$$

where

$$
h_{s}(x)=\sum_{1 \leq j \leq s} \frac{j x^{j}}{1-x^{j}}
$$


Similarly, using (2.3), (2.6) and an argument developed by Wilf [15], one can deduce that

$$
1+\sum_{n=1}^{\infty} p(n) x^{n} \mathbb{E}\left(y^{Y_{s, n}}\right)=g(x) \prod_{1 \leq j \leq s}\left(1+(y-1) x^{j}\right)
$$

and, in the same way, that

$$
\sum_{n=1}^{\infty} p(n) x^{n} \mathbb{E}\left(Y_{s, n}\right)=g(x) \frac{1-x^{[s]+1}}{1-x} .
$$

It is shown in [11] that, for $s=t \sqrt{6 n} / \pi, 0<t<\infty$,

$$
\mathbb{E}\left(Y_{s, n}\right) \sim\left(1-e^{-t}\right) \frac{\sqrt{6 n}}{\pi}, \quad n \rightarrow \infty .
$$

Finally, we notice that the asymptotic behavior of $Z_{s, n}$, defined by (2.5), is studied in detail by Fristedt [5]. He assumed that $s / \sqrt{n} \rightarrow 0$ as $n \rightarrow \infty$ and showed that $Z_{s, n}$, appropriately normalized, converges in distribution to a doubly exponential (extreme value) distributed random variable. A law of large numbers for $Z_{s, n}$ is also proved.

We now proceed with the description of the sampling procedures introduced by Corteel et al. [3]. We remind that they are three two-step procedures that combine the outcomes of two experiments. Therefore, they lead to three different product probability spaces (see e.g. [7; Chapter 1.6]). Since in each procedure we first sample uar a partition $\lambda \in \Lambda(n)$, the probability space on $\Lambda(n)$, defined in the Introduction, is included in each product space. The second steps of sampling are, however, different and therefore, for each different procedure we obtain a different product space and different product probability measure. In what follows next we adopt the common notation $\mathbb{P}($.$) for the$ product probability measure of each sampling procedure, use notation $\sigma_{n, j}$ for the size of the randomly-chosen part in procedure $j(j=1,2,3)$ and follow the concept of a product space developed in [7; Chapter 1.6]. We describe the procedures in terms of events $\{\lambda \in \Lambda(n)\},\left\{\sigma_{n, j} \leq s\right\}(s \geq 1)$ and their set product $\{\lambda \in \Lambda(n)\} \times\left\{\sigma_{n, j} \leq s\right\}$.

Procedure 1. Given a partition $\lambda \in \Lambda(n)$ chosen uar (step 1), we select a part uar among all $Y_{n}$ different parts of $\lambda$ (step 2). Hence by the product measure formula [7; Chapter 1.6] and (2.6),

$$
\mathbb{P}\left(\{\lambda \in \Lambda(n)\} \times\left\{\sigma_{n, 1} \leq s\right\}\right)=\operatorname{Pr}(\lambda \in \Lambda(n))\left(\frac{Y_{s, n}}{Y_{n}}\right)=\left(\frac{1}{p(n)}\right)\left(\frac{Y_{s, n}}{Y_{n}}\right) .
$$

Summation over all $\lambda \in \Lambda(n)$ yields

$$
\mathbb{P}\left(\sigma_{n, 1} \leq s\right)=\mathbb{E}\left(\frac{Y_{s, n}}{Y_{n}}\right) .
$$

Procedure 2. Given a partition $\lambda \in \Lambda(n)$ chosen uar (step 1), we select a part of it with the probability proportional to its size and multiplicity (step 2). 
Recalling definition (2.4) of the random variable $X_{s, n}$, we obtain in a similar way that

$$
\mathbb{P}\left(\{\lambda \in \Lambda(n)\} \times\left\{\sigma_{n, 2} \leq s\right\}\right)=\left(\frac{1}{p(n)}\right)\left(\frac{X_{s, n}}{n}\right)
$$

and

$$
\mathbb{P}\left(\sigma_{n, 2} \leq s\right)=\frac{1}{n} \mathbb{E}\left(X_{s, n}\right) .
$$

Procedure 3. Given a partition $\lambda \in \Lambda(n)$ chosen uar (step 1), we select a part of it uar among all $Z_{n}$ parts in $\lambda$ (without any bias, step 2), i.e.,

$$
\mathbb{P}\left(\{\lambda \in \Lambda(n)\} \times\left\{\sigma_{n, 3} \leq s\right\}\right)=\left(\frac{1}{p(n)}\right)\left(\frac{Z_{s, n}}{Z_{n}}\right),
$$

where $Z_{s, n}$ is defined by (2.5), and thus

$$
\mathbb{P}\left(\sigma_{n, 3} \leq s\right)=\mathbb{E}\left(\frac{Z_{s, n}}{Z_{n}}\right) .
$$

Remark. Each partition $\lambda \in \Lambda(n)$ has a unique graphical representation called Ferrers diagram [2; Chapter 1.3]. It is obtained as follows. We use the notation $\lambda_{k}$ to denote the $k$ th largest part of $\lambda$ for $k$ a positive integer; if the number of parts $Z_{n}$ of $\lambda$ is $<k$, then $\lambda_{k}=0$. The Ferrers diagram illustrates (1.1) by a two-dimensional array of dots, composed by $\lambda_{1}$ dots in the first (most left) row, $\lambda_{2}$ dots in the second row, $\ldots, \lambda_{Z_{n}}$ dots in the last $Z_{n}$ th row. Therefore, a Ferrers diagram may be considered as a union of disjoint blocks (rectangles) of dots with base $j$ and height $\alpha_{j}^{(n)}$ (the multiplicity of part $j$ ). In this way, the sampling probability in Procedure 2 is proportional to the area of the block to which the chosen part belongs.

In order to make a comparison between the asymptotic behavior of the typical part size $\sigma_{n, j}$ (see next section) and its typical multiplicity $\mu_{n, j}$ for procedure $j(j=1,2,3)$, we also provide the reader with some results, obtained by Corteel et al. [3]:

$$
\begin{gathered}
\lim _{n \rightarrow \infty} \mathbb{P}\left(\mu_{n, 1}=m\right)=\frac{1}{m(m+1)}, \quad m \geq 1, \\
\lim _{n \rightarrow \infty} \mathbb{P}\left(\mu_{n, 2}=m\right)=\frac{6(2 m+1)}{\pi^{2} m(m+1)^{2}}, \quad m \geq 1
\end{gathered}
$$

and

$$
\lim _{n \rightarrow \infty} \mathbb{P}\left(\frac{2 \log \mu_{n, 3}}{\log n} \leq t\right)=t, \quad 0<t<1
$$

\section{Statement of the Main Results and Brief De- scription of the Method of Proof}

Among other important results on random integer partitions Fristedt [5; p. 712] has proved the following limit theorem. 
Theorem 1 Let $0<t<1$. Then, we have

$$
\lim _{n \rightarrow \infty} \mathbb{P}\left(\frac{2 \log \sigma_{n, 3}}{\log n} \leq t\right)=t .
$$

Remark. A comparison between this result and (2.19) shows that both $\mu_{n, 3}$ and $\sigma_{n, 3}$, normalized in the same manner, have one and the same limiting distribution as $n \rightarrow \infty$. Moreover, these results imply that the proportion of part sizes not greater than $n^{t / 2}$ and the proportion of parts whose multiplicity is $\leq n^{t / 2}$ are both approximately equal to $t \in(0,1)$ as $n \rightarrow \infty$.

The main results of this paper are devoted to sampling procedures 1 and 2 . In the next sections we prove the following limit theorems for the randomlychosen part size $\sigma_{n, j}, j=1,2$, of a random integer partition.

Theorem 2 Let $0<t<\infty$. Then, we have

$$
\lim _{n \rightarrow \infty} \mathbb{P}\left(\frac{\pi \sigma_{n, 1}}{\sqrt{6 n}} \leq t\right)=1-e^{-t} .
$$

Theorem 3 Let $0<t<\infty$. Then, we have

$$
\lim _{n \rightarrow \infty} \mathbb{P}\left(\frac{\pi \sigma_{n, 2}}{\sqrt{6 n}} \leq t\right)=\frac{6}{\pi^{2}} \int_{0}^{t} \frac{u}{e^{u}-1} d u .
$$

Remark. The limiting distribution in Theorem 3 is expressed in terms of a Debye function (see e.g. [1; Section 27.1]). We recall that

$$
\int_{0}^{\infty} \frac{u}{e^{u}-1} d u=\frac{\pi^{2}}{6}
$$

We also notice that Theorems 2 and 3 show that, for sampling procedures 1 and 2, the typical size of the randomly-chosen part is of order const. $\sqrt{n}$, while (2.17) and (2.18) imply that its multiplicity is much smaller and approaches a discrete random variable as $n \rightarrow \infty$.

The rest of the paper contains the proofs of Theorems 2 and 3 . In the proof of Theorem 2 we use Fristedt's conditioning device [5], which allows us to transfer probability distributions of linear combinations of the multiplicities

$\alpha_{j}^{(n)}$ into conditional distributions of the corresponding linear combinations of independent geometrically distributed random variables. This allows us to approximate the expectations in (2.14) and (2.16) by the ratios of the expected values of the corresponding random variables. The proof of Theorem 3 is based on a Cauchy integral stemming from (2.11), Hardy-Ramanujan's formula (1.2) and Hayman's theorem for estimating coefficients of admissible power series [9] (see also [4; Chapter VIII.5]).

\section{Proof of Theorem 2}

We base our proof on (2.14) and asymptotic equivalences (2.7) and (2.13). We follow the argument given in the proof of Theorem 3 of [3]. To replace the 
expectation in the right-hand side of (2.14) by the ratio $\mathbb{E}\left(Y_{s, n}\right) / \mathbb{E}\left(Y_{n}\right)$, we need to study how unlikely is the event

$$
A_{n}=\left\{\lambda \in \Lambda(n):\left|\frac{\pi Y_{n}(\lambda)}{\sqrt{6 n}}-1\right|>\epsilon\right\}, \quad \epsilon>0 .
$$

Using Fristedt's method [5], Corteel et al. [3] showed that

$$
\operatorname{Pr}\left(A_{n}\right) \leq e^{-c \sqrt{n}}, \quad c=c(\epsilon)>0 .
$$

Remark. Fristedt's approach [5] is based on the identity

$$
\operatorname{Pr}\left(\alpha_{j}^{(n)}=m_{j}, j=1, \ldots, n\right)=\operatorname{Pr}\left(\gamma_{j}=m_{j}, j=1, \ldots, n \mid \sum_{j \geq 1} j \gamma_{j}=n\right),
$$

where $\left\{\gamma_{j}\right\}_{j \geq 1}$ is a sequence of independent geometrically distributed random variables, whose distribution is given by

$$
\operatorname{Pr}\left(\gamma_{j}=k\right)=\left(1-q^{j}\right) q^{j k}, \quad k=0,1, \ldots
$$

and $\left\{m_{j}\right\}_{j \geq 1}$ are non-negative integers. Eq. (4.2) holds for every fixed $q \in(0,1)$. It is natural to take $q$ so that $\operatorname{Pr}\left(\sum_{j \geq 1} j \gamma_{j}=n\right)$ is as large as possible. Fristedt's almost optimal choice for $q$ is $q=e^{-\pi / \sqrt{6 n}}$. Then, the bound in (4.1) is easily obtained using this value of $q$.

Next, we represent the probability in (2.14) in the following way

$$
\mathbb{P}\left(\sigma_{n, 1} \leq s\right)=\mathbb{E}\left(\frac{Y_{s, n}}{Y_{n}} I_{A_{n}^{c}}\right)+\mathbb{E}\left(\frac{Y_{s, n}}{Y_{n}} I_{A_{n}}\right),
$$

where $I_{A_{n}}$ and $I_{A_{n}^{c}}$ denote the indicators of events $A_{n}$ and $A_{n}^{c}$, respectively. Since, for any $\lambda \in A_{n}^{c}$,

$$
\frac{\pi}{\sqrt{6 n}(1+\epsilon)}<\frac{1}{Y_{n}}<\frac{\pi}{\sqrt{6 n}(1-\epsilon)}
$$

if $0<\epsilon<1$, the first summand in (4.3) is estimated by

$$
\begin{aligned}
& \mathbb{E}\left(\frac{Y_{s, n}}{Y_{n}} I_{A_{n}^{c}}\right)=\frac{\pi}{\sqrt{6 n}}(1+O(\epsilon)) \mathbb{E}\left(Y_{s, n} I_{A_{n}^{c}}\right) \\
& =\frac{\pi}{\sqrt{6 n}}(1+O(\epsilon))\left(\mathbb{E}\left(Y_{s, n}\right)-\mathbb{E}\left(Y_{s, n} I_{A_{n}}\right)\right) .
\end{aligned}
$$

Clearly, with probability 1 , we have $Y_{s, n} \leq n$. Hence, using (4.1), we obtain

$$
\mathbb{E}\left(Y_{s, n} I_{A_{n}}\right)=O\left(n \operatorname{Pr}\left(A_{n}\right)\right)=O\left(n e^{-c \sqrt{n}}\right) .
$$

Then, for $s=t \sqrt{6 n} / \pi$, by (2.13) and (4.4),

$$
\begin{aligned}
& \mathbb{E}\left(\frac{Y_{s, n}}{Y_{n}} I_{A_{n}^{c}}\right)=\frac{\pi}{\sqrt{6 n}}(1+O(\epsilon))\left(\frac{\sqrt{6 n}}{\pi}\left(1-e^{-t}\right)\right)(1+o(1)) \\
& +O\left(n e^{-c \sqrt{n}}\right)=1-e^{-t}+O(\epsilon)+o(1)+O\left(n e^{-c \sqrt{n}}\right)
\end{aligned}
$$


The second term in the right-hand side of (4.3) is easily estimated using (4.1) since it is not greater than $\operatorname{Pr}\left(A_{n}\right)$. Consequently, (4.3) becomes

$$
\mathbb{P}\left(\sigma_{n, 1} \leq s\right)=\mathbb{P}\left(\frac{\pi \sigma_{n, 1}}{\sqrt{6 n}} \leq t\right)=1-e^{-t}+O(\epsilon)+o(1) .
$$

Letting $n \rightarrow \infty$ and then $\epsilon \rightarrow 0$, we obtain the required result.

\section{Some Remarks on Meinardus Theorem on Weighted Partitions and Hayman Admissibility}

This section presents a brief introduction to the analytic combinatorics background needed for the proof of Theorem 3. The starting point in it is eq. (2.15). It requires an asymptotic estimate for $\mathbb{E}\left(X_{s, n}\right)$. By (2.11), the coefficient $p(n) \mathbb{E}\left(X_{s, n}\right)$ of $x^{n}$ can be expressed by a Cauchy integral whose integrand is $g(x) h_{s}(x)$ (see also (2.12) ). Its behavior heavily depends on the analytic properties of the partition generating function $g(x)$ whose infinite product representation (2.1) shows that its main singularity is at $x=1$ (see [2; Chapter 5]). If the integrand of the Cauchy integral was only $g(x)$, then a properly chosen contour of integration and a proper asymptotic method (the Hardy-Ramanujan circle method or the saddle-point method) would yield Hardy-Ramanujan asymptotic formula (1.2). It has been subsequently generalized in various directions most notably by Meinardus [10] (see also [2; Chapter 6]) who obtained the asymptotic of the Taylor coefficients of infinite products of the form

$$
\prod_{k=1}^{\infty}\left(1-x^{k}\right)^{-b_{k}}
$$

under certain general assumptions on the sequence of non-negative numbers $\left\{b_{k}\right\}_{k \geq 1}$. Meinardus approach is based on considering the Dirichlet generating series

$$
D(z)=\sum_{k=1}^{\infty} b_{k} k^{-z}, \quad z=u+i v .
$$

Below we briefly describe Meinardus assumptions avoiding their precise statements as well as some extra notations and concepts. The first assumption $\left(M_{1}\right)$ specifies the domain $\mathcal{H}=\left\{z: u \geq-C_{0}\right\}, 0<C_{0}<1$, in the complex plane, in which $D(z)$ has an analytic continuation. The second one $\left(M_{2}\right)$ is related to the asymptotic behavior of $D(z)$, whenever $|v| \rightarrow \infty$. A function of the complex variable $z$ which is bounded by $O\left(|\Im(z)|^{C_{1}}\right), 0<C_{1}<\infty$, in certain domain of the complex plane is called function of finite order. Meinardus second condition $\left(M_{2}\right)$ requires that $D(z)$ is of finite order in the whole domain $\mathcal{H}$. Finally, the Meinardus third condition $\left(M_{3}\right)$ implies a bound on the ordinary generating function of the sequence $\left\{b_{k}\right\}_{k \geq 1}$. It can be stated in a way simpler than the 
Meinardus original expression by the inequality

$$
\sum_{k=1}^{\infty} b_{k} e^{-k \omega} \sin ^{2}(\pi k u) \geq C_{2} \omega^{-\epsilon_{1}}, \quad 0<\frac{\omega}{2 \pi}<|u|<\frac{1}{2},
$$

for sufficiently small $\omega$ and some constants $C_{2}, \epsilon_{1}>0\left(C_{2}=C_{2}\left(\epsilon_{1}\right)\right)$ (see [6; p. $310])$.

It is known that Euler partition generating function $g(x)$ (which is obviously of the form (5.1)) satisfies the Meinardus scheme of conditions $\left(M_{1}\right)-\left(M_{3}\right)$ (see e.g. [2; Theorem 6.3]).

In the asymptotic analysis of the Cauchy integral stemming from (2.11) we apply the saddle-point method using Hayman admissibility theory [9]; see also [4; Chapter VIII.5]. Hayman studied a wide class of power series satisfying a set of relatively mild conditions and established general formulas for the asymptotic order of their coefficients. To present Hayman's idea and show how it can be applied in the proof of our Theorem 3, we need to introduce some auxiliary notations.

We consider here a function $G(x)=\sum_{n=1}^{\infty} G_{n} x^{n}$ that is analytic for $|x|<$ $\rho, 0<\rho<\infty$. For $0<r<\rho$, we let

$$
\begin{gathered}
a(r)=r \frac{G^{\prime}(r)}{G(r)}, \\
b(r)=r \frac{G^{\prime}(r)}{G(r)}+r^{2} \frac{G^{\prime \prime}(r)}{G(r)}-r^{2}\left(\frac{G^{\prime}(r)}{G(r)}\right) .
\end{gathered}
$$

In the statement of the Hayman's result we use the terminology given in [4; Chapter VIII.5]. We assume that $G(x)>0$ for $x \in\left(R_{0}, \rho\right) \subset(0, \rho)$ and satisfies the following three conditions.

Capture condition. $\lim _{r \rightarrow \rho} a(r)=\infty$ and $\lim _{r \rightarrow \rho} b(r)=\infty$.

Locality condition. For some function $\delta=\delta(r)$ defined over $\left(R_{0}, \rho\right)$ and satisfying $0<\delta<\pi$, one has

$$
G\left(r e^{i \theta}\right) \sim G(r) e^{i \theta a(r)-\theta^{2} b(r) / 2}
$$

as $r \rightarrow \rho$, uniformly for $|\theta| \leq \delta(r)$.

Decay condition.

$$
G\left(r e^{i \theta}\right)=o\left(\frac{G(r)}{\sqrt{b(r)}}\right)
$$

as $r \rightarrow \rho$, uniformly for $\delta(r) \leq \theta<\pi$.

Hayman Theorem. Let $G(x)$ be Hayman admissible function and $r=r_{n}$ be the unique solution in the interval $\left(R_{0}, \rho\right)$ of the equation

$$
a(r)=n .
$$

Then the Taylor coefficients of $G(x)$ satisfy, as $n \rightarrow \infty$,

$$
G_{n} \sim \frac{G\left(r_{n}\right)}{r_{n}^{n} \sqrt{2 \pi b\left(r_{n}\right)}}
$$


with $b\left(r_{n}\right)$ given by (5.4).

The proof of Theorem 3 (see next section) is divided into two parts.

A) Proof of Hayman admissibility for $g(x)$. (2.11).

B) Obtaining an asymptotic estimate for the Cauchy integral stemming from

\section{Proof of Theorem 3}

Part A.

First we need to show how Hayman's theorem can be applied to find the asymptotic behavior of the Taylor coefficients of the partition generating function $g(x)$. Since in (2.1) we have $b_{k}=1, k \geq 1$, the Dirichlet generating series (5.2) is $D(z)=\zeta(z)$, where $\zeta$ denotes the Riemann zeta function. We set in (5.3) and (5.4) $r=r_{n}=e^{-d_{n}}, d_{n}>0$, where $d_{n}$ is the unique solution of the equation

$$
a\left(e^{-d_{n}}\right)=n .
$$

(6.1) is an obvious modification of (5.5).) Granovsky et al. [6] showed that the first two Meinardus conditions imply that the unique solution of (6.1) has the following asymptotic expansion:

$$
d_{n}=\sqrt{\zeta(2) / n}+\frac{\zeta(0)}{2 n}+O\left(n^{-1-\beta}\right)=\frac{\pi}{\sqrt{6 n}}-\frac{1}{4 n}+O\left(n^{-1-\beta}\right),
$$

where $\beta>0$ is fixed constant (here we have also used that $\zeta(0)=-1 / 2$; see $[1$; Chapter 23.2]). We also notice that (5.4) and (6.2) impliy that

$$
b\left(e^{-d_{n}}\right)=2 \zeta(2) d_{n}^{-3}+O\left(d_{n}^{-2}\right) \sim \frac{\pi^{2}}{3} d_{n}^{-3} \sim \frac{2 \sqrt{6}}{\pi} n^{3 / 2}
$$

(see [12; Lemma 2.2] with $D(z)=\zeta(z)$ ). Hence, by (6.1) and (6.3), $a\left(e^{-d_{n}}\right) \rightarrow \infty$ and $b\left(e^{-d_{n}}\right) \rightarrow \infty$ as $n \rightarrow \infty$, that is, Hayman's "capture" condition is satisfied with $r=r_{n}=e^{-d_{n}}$. To show next that Hayman's "decay" condition is satisfied by $g(x)$ we set

$$
\delta_{n}=\frac{d_{n}^{4 / 3}}{\Omega(n)}=\frac{\pi^{4 / 3}}{(6 n)^{2 / 3} \Omega(n)}\left(1+O\left(\frac{1}{\sqrt{n}}\right)\right)
$$

with $d_{n}$ given by (6.2), where $\Omega(n) \rightarrow \infty$ as $n \rightarrow \infty$ arbitrarily slowly. We can apply now an estimate for $\left|g\left(e^{-d_{n}+i \theta}\right)\right|$ established in a general form in [12; Lemma 2.4] using all three Meinardus conditions. It states that there are two positive constants $c_{0}$ and $\epsilon_{0}$, such that, for sufficiently large $n$,

$$
\left|g\left(e^{-d_{n}+i \theta}\right)\right| \leq g\left(e^{-d_{n}}\right) e^{-c_{0} d_{n}^{-\epsilon_{0}}}
$$

uniformly for $\delta_{n} \leq|\theta|<\pi$. This, in combination with (6.3), implies that $\left|g\left(e^{-d_{n}+i \theta}\right)\right|=o\left(g\left(e^{-d_{n}}\right) / \sqrt{b\left(e^{-d_{n}}\right)}\right)$ uniformly in the same range for $\theta$, which 
is just Hayman's "decay" condition. Finally, by Lemma 2.3 of [12], established using Meinardus conditions $\left(M_{1}\right)$ and $\left(M_{2}\right)$, Hayman's "locality" condition is also satisfied by $g(x)$. In fact, this lemma implies in the particular case $D(z)=$ $\zeta(z)$ that

$$
e^{-i \theta n} \frac{g\left(e^{-d_{n}+i \theta}\right)}{g\left(e^{-d_{n}}\right)}=e^{-\theta^{2} b\left(e^{-d_{n}}\right) / 2}\left(1+O\left(1 / \Omega^{3}(n)\right)\right.
$$

uniformly for $|\theta| \leq \delta_{n}$, where $b\left(e^{-d_{n}}\right)$ and $\delta_{n}$ are determined by (6.3) and (6.4), respectively. Hence all conditions of Hayman's theorem hold and we can apply it with $G_{n}=p(n), G(x)=g(x), r_{n}=e^{-d_{n}}$ and $\rho=1$ to find that

$$
p(n) \sim \frac{e^{n d_{n}} g\left(e^{-d_{n}}\right)}{\sqrt{2 \pi b\left(e^{-d_{n}}\right)}}, \quad n \rightarrow \infty .
$$

Remark. To show that formula (6.7) yields (1.2), one has to replace (6.2) and (6.3) in the right hand side of (6.7). The asymptotic of $g\left(e^{-d_{n}}\right)$ is determined by a general lemma due to Meinardus [10] (see also [2; Lemma 6.1]). Since $\zeta(0)=-1 / 2$ and $\zeta^{\prime}(0)=-\frac{1}{2} \log (2 \pi)$ (see [1; Chapter 23.2]), in the particular case of $g\left(e^{-d_{n}}\right)$ this lemma implies that

$$
\begin{aligned}
& g\left(e^{-d_{n}}\right)=\exp \left(\zeta(2) d_{n}^{-1}-\zeta(0) \log d_{n}+\zeta^{\prime}(0)+O\left(d_{n}^{c_{1}}\right)\right) \\
& =\exp \left(\frac{\pi^{2}}{6 d_{n}}+\frac{1}{2} \log d_{n}-\frac{1}{2} \log (2 \pi)+O\left(d_{n}^{c_{1}}\right)\right), \quad n \rightarrow \infty,
\end{aligned}
$$

where $0<c_{1}<1$. The rest of the computation leading to (1.2) is based on simple algebraic manipulations and cancellations.

Part B.

We are now ready to apply Cauchy coefficient formula to (2.11). We use the circle $x=e^{-d_{n}+i \theta},-\pi<\theta \leq \pi$, as a contour of integration and obtain

$$
p(n) \mathbb{E}\left(X_{s, n}\right)=\frac{e^{n d_{n}}}{2 \pi} \int_{-\pi}^{\pi} g\left(e^{-d_{n}+i \theta}\right) h_{s}\left(e^{-d_{n}+i \theta}\right) e^{-i \theta n} d \theta .
$$

Then, we break up the range of integration as follows:

$$
p(n) \mathbb{E}\left(X_{s, n}\right)=J_{1}(s, n)+J_{2}(s, n),
$$

where

$$
\begin{gathered}
J_{1}(s, n)=\frac{e^{n d_{n}}}{2 \pi} \int_{-\delta_{n}}^{\delta_{n}} g\left(e^{-d_{n}+i \theta}\right) h_{s}\left(e^{-d_{n}+i \theta}\right) d \theta, \\
J_{2}(s, n)=\frac{e^{n d_{n}}}{2 \pi} \int_{\delta_{n}<|\theta| \leq \pi} g\left(e^{-d_{n}+i \theta}\right) h_{s}\left(e^{-d_{n}+i \theta}\right) d \theta
\end{gathered}
$$

and $\delta_{n}$ is defined by (6.4). 
To estimate $J_{2}(s, n)$, for $s=t \sqrt{6 n} / \pi, 0<t<\infty$, we notice that by the definition of Riemann integrals and (6.2),

$$
\begin{aligned}
& \left|h_{s}\left(e^{-d_{n}+i \theta}\right)\right| \leq \sum_{1 \leq j \leq s} \frac{j e^{-j d_{n}}}{\left|1-e^{-j d_{n}+i j \theta}\right|} \\
& \leq d_{n}^{-2} \sum_{d_{n} \leq j d_{n} \leq s d_{n}} \frac{j d_{n} e^{-j d_{n}}}{1-e^{-j d_{n}}} d_{n} \\
& \sim d_{n}^{-2} \int_{0}^{t} \frac{u e^{-u}}{1-e^{-u}} d u=O\left(d_{n}^{-2}\right)=O(n) .
\end{aligned}
$$

Combining (6.2), (6.3), (6.5), 6.7), 6.10) and (6.11), we obtain

$$
\begin{aligned}
& \left|J_{2}(s, n)\right| \leq \frac{e^{n d_{n}}}{2 \pi} \int_{\delta_{n}<|\theta| \leq \pi}\left|g\left(e^{-d_{n}+i \theta}\right) h_{s}\left(e^{-d_{n}+i \theta}\right)\right| d \theta \\
& =O\left(e^{n d_{n}} g\left(e^{-d_{n}}\right) n e^{-c_{0} d_{n}^{-\epsilon_{0}}}\right)=O\left(\frac{e^{n d_{n}} g\left(e^{-d_{n}}\right)}{\sqrt{b\left(e^{-d_{n}}\right)}} n^{1+3 / 4} e^{-c_{0} d_{n}^{-\epsilon_{0}}}\right) \\
& =O\left(p(n) n^{7 / 4} e^{-c_{0} d_{n}^{-\epsilon_{0}}}\right)=O\left(p(n) n^{7 / 4} e^{-c_{2} n^{\epsilon_{0} / 2}}\right)=o(n p(n)),
\end{aligned}
$$

where $c_{2}>0$.

The estimate of $J_{1}(s, n)$ follows from Hayman's "locality" condition (6.6). We also need to expand $h_{s}$ by Taylor formula in the following way:

$$
\begin{aligned}
& h_{s}\left(e^{-d_{n}+i \theta}\right)=h_{s}\left(e^{-d_{n}}\right)+O\left(\left.|\theta| \frac{d}{d x} h_{s}(x)\right|_{x=e^{-d_{n}}}\right) \\
& =h_{s}\left(e^{-d_{n}}\right)+O\left(\left.\delta_{n} \frac{d}{d x} h_{s}(x)\right|_{x=e^{-d_{n}}}\right) .
\end{aligned}
$$

For $s=t \sqrt{6 n} / \pi$, we can consider, as previously, the sum representing $h_{s}\left(e^{-d_{n}}\right)$ as a Riemann sum. So, we can replace it by the corresponding integral. Thus, by (2.12) and (6.2), we have

$$
\begin{aligned}
& h_{s}\left(e^{-d_{n}}\right)=d_{n}^{-2} \sum_{d_{n} \leq j d_{n} \leq t \sqrt{6 n} d_{n} / \pi} \frac{j d_{n} e^{-j d_{n}}}{1-e^{-j d_{n}}} d_{n} \\
& \sim d_{n}^{-2} \int_{d_{n}}^{\frac{\sqrt{6 n}}{\pi} d_{n} t} \frac{u e^{-u}}{1-e^{-u}} d u=\frac{6 n}{\pi^{2}} \int_{0}^{t} \frac{u}{e^{u}-1} d u+O(\sqrt{n})
\end{aligned}
$$

since $d_{n}^{-2}=6 n / \pi^{2}+O(\sqrt{n})$. In the same way we can estimate the first derivative of $h_{s}$ :

$$
\begin{aligned}
& \left.\frac{d}{d x} h_{s}(x)\right|_{x=e^{-d_{n}}}=\sum_{1 \leq j \leq s} \frac{j^{2} e^{(j-1) d_{n}}}{\left(1-e^{-j d_{n}}\right)^{2}} \sim d_{n}^{-3} \sum_{d_{n} \leq j d_{n} \leq t \sqrt{6 n} d_{n} / \pi} \frac{\left(j d_{n}\right)^{2} e^{-j d_{n}}}{\left(1-e^{-j d_{n}}\right)^{2}} d_{n} \\
& \sim d_{n}^{-3} \int_{0}^{t} \frac{u^{2} e^{-u}}{\left(1-e^{-u}\right)^{2}} d u=O\left(n^{3 / 2}\right) .
\end{aligned}
$$


Hence, by (6.4), the error term in (6.13) becomes

$$
O\left(\left.\delta_{n} \frac{d}{d x} h_{s}(x)\right|_{x=e^{-d_{n}}}\right)=O\left(n^{5 / 6} / \Omega(n)\right)
$$

and therefore, uniformly for $|\theta| \leq \delta_{n}$,

$$
h_{s}\left(e^{-d_{n}+i \theta}\right)=\frac{6 n}{\pi^{2}} \int_{0}^{t} \frac{u}{e^{u}-1} d u+o(n) .
$$

Inserting this estimate and (6.6) into (6.9) and applying the asymptotic of the partition function $p(n)$ from (6.7), we obtain

$$
\begin{aligned}
J_{1}(s, n)=\frac{e^{n d_{n}} g\left(e^{-d_{n}}\right)}{2 \pi}\left(\int_{-\delta_{n}}^{\delta_{n}} e^{-\theta^{2} b\left(e^{-d_{n}}\right) / 2}\left(1+O\left(1 / \Omega^{3}(n)\right) d \theta\right)\right. \\
\times\left(\frac{6 n}{\pi^{2}} \int_{0}^{t} \frac{u}{e^{u}-1} d u+o(n)\right) \\
\sim \frac{e^{n d_{n}} g\left(e^{-d_{n}}\right)}{\sqrt{b\left(e^{-d_{n}}\right)} 2 \pi}\left(\int_{-\delta_{n} \sqrt{b\left(e^{-d_{n}}\right)}}^{\delta_{n} \sqrt{b\left(e^{-d_{n}}\right)}} e^{-y^{2} / 2} d y\right)\left(\frac{6 n}{\pi^{2}} \int_{0}^{t} \frac{u}{e^{u}-1} d u\right) \\
\sim \frac{e^{n d_{n}} g\left(e^{-d_{n}}\right)}{\sqrt{b\left(e^{-d_{n}}\right)} 2 \pi}\left(\int_{-\infty}^{\infty} e^{-y^{2} / 2} d y\right)\left(\frac{6 n}{\pi^{2}} \int_{0}^{t} \frac{u}{e^{u}-1} d u\right) \\
=\frac{e^{n d_{n}} g\left(e^{-d_{n}}\right)}{\sqrt{2 \pi b\left(e^{-d_{n}}\right)}}\left(\frac{6 n}{\pi^{2}} \int_{0}^{t} \frac{u}{e^{u}-1} d u\right) \\
\sim p(n)\left(\frac{6 n}{\pi^{2}} \int_{0}^{t} \frac{u}{e^{u}-1} d u\right),
\end{aligned}
$$

where for the second asymptotic equivalence we have used (6.3) and (6.4) in order to get

$$
\delta_{n} \sqrt{b\left(e^{-d_{n}}\right)} \sim \frac{\pi^{5 / 6} \sqrt{2}}{6^{1 / 6} \Omega(n)} n^{1 / 12} \rightarrow \infty
$$

if $\Omega(n) \rightarrow \infty$ as $n \rightarrow \infty$ not too fast, so that $\frac{n^{1 / 12}}{\Omega(n)} \rightarrow \infty$. It is now clear that, for $s=t \sqrt{6 n} / \pi$, (6.8)-(6.10), (6.12) and (6.14) yield

$$
p(n) \mathbb{E}\left(X_{s, n}\right)=p(n) \frac{6 n}{\pi^{2}} \int_{0}^{t} \frac{u}{e^{u}-1} d u+o(n p(n))
$$

and therefore

$$
\mathbb{E}\left(X_{s, n}\right) \sim \frac{6 n}{\pi^{2}} \int_{0}^{t} \frac{u}{e^{u}-1} d u .
$$

The result of Theorem 3 follows immediately from (2.15).

\section{Acknowledgements}

I am grateful the referee for carefully reading the paper and for his helpful comments. 


\section{References}

[1] Abramovitz, M., Stegun, I.A.: Handbook of Mathemathical Functions with Formulas, Graphs and Mathematical Tables. Dover Publ. Inc., New York (1965).

[2] Andrews, G.E.: The Theory of Partitions. Encyclopedia Math. Appl. 2. Addison-Wesley, Reading, MA (1976).

[3] Corteel, S., Pittel, B., Savage, C.D., Wilf, H.S.: On the multiplicity of parts in a random partition. Random Stuct. Alg. 14, 185-197(1999).

[4] Flajolet, P., Sedgewick, R.: Analytic Combinatorics. Cambridge University Press, Cambridge (2009).

[5] Fristedt, B.: The structure of random partitions of large integers. Trans. Amer. Math. Soc. 337, 703-735 (1993).

[6] Granovsky, B., Stark, D., Erlihson, M.: Meinardus theorem on weighted partitions: Extensions and a probabilistic proof. Adv. Appl. Math. 41, 307328 (2008).

[7] Grimmett, G., Stirzaker, D.: Probability and Random Processes. Oxford University Press, Oxford (2001).

[8] Hardy, G.H., Ramanujan, S.: Asymptotic formulae in combinatory analysis. Proc. London Math. Soc. 17(2), 75-115 (1918).

[9] Hayman, W.K.: A generalization of Stirling's formula. J. Reine Angew. Math. 196, 67-95 (1956).

[10] Meinardus, G.: Asymptotische Aussagen über Partitionen. Math. Z. 59, 388-398 (1954).

[11] Mutafchiev, L.: Large distinct part sizes in a random integer partition. Acta Math. Hungar. 87, 47-69 (2000).

[12] Mutafchiev, L.: The size of the largest part of random weighted partitions of large integers. Combinatorics Probab. Comput. 22, 433-454 (2013).

[13] Rademacher, H.: On the partition function $p(n)$. Proc. London Math. Soc. 43, 241-254 (1937).

[14] Sachkov, V.N.: Combinatorial Methods in Discrete Mathematics. Encyclopedia Math. Appl. 55. Cambridge University Press, Cambridge (1999).

[15] Wilf, H.: Three problems in combinatorial analysis. J. Combin. Theory Ser. A 35, 199-207 (1983). 